J. Klin. Endokrinol. Stoffw. 2019 • 12:50-54 https://doi.org/10.1007/s41969-019-0063-x Online publiziert: 5. Juni 2019

(c) Der/die Autor(en) 2019
Karin Amrein ${ }^{1,2} \cdot$ Harald Dobnig ${ }^{1}$

'Schilddrüsen/Endokrinologie/Osteoporose Institut Dobnig, Graz, Österreich

${ }^{2}$ Abteilung für Endokrinologie und Diabetologie, Medizinische Universität Graz, Graz, Österreich

\title{
Primärer Hyperparathyreoidismus
}

\section{Drei spannende Fallberichte und Neuigkeiten zu Diagnose und Therapie}

\section{Einleitung}

Der primäre Hyperparathyreoidismus (pHPT) ist eine der häufigsten endokrinen Erkrankungen und betrifft weitaus mehr Frauen als Männer. Dennoch gibt es häufig Verzögerungen in der Diagnostik und dem Anbieten einer Therapie [1]. Mit steigender Anzahl von RoutineKalziumbestimmungen hat sich in den letzten Jahrzehnten ein deutlicher Shift von ursprünglich weitgehend symptomatischen $\mathrm{zu}$ meist asymptomatischen Formen gezeigt, da die Diagnose nun wesentlich häufiger sehr früh gestellt wird [2]. Die „Stein-, Bein- und Magenpein" sehen wir daher nur noch selten. Heutzutage bestehen häufig unspezifische Symptome wie vermehrte Müdigkeit, eingeschränkte Leistungsfähigkeit und arterielle Hypertonie, aber auch Störungen der Muskelfunktion und Nierensteine werden bei „asymptomatischen" Patienten beschrieben [3, 4]. Selten kommt der pHPT im Rahmen von Syndromen wie der multiplen endokrinen Neoplasie (MEN; 1, 2A und 4) oder dem pHPT-Jaw-TumorSyndrom vor [5-7]. Zumeist ist ein singuläres Adenom für die Mehrsekretion verantwortlich, in ca. $5 \%$ sind es zwei oder mehr Adenome, in ca. 5\% besteht eine Hyperplasie, und Nebenschilddrüsenkarzinome sind eine Rarität [8]. Eine wichtige und manchmal gar nicht so triviale Differenzialdiagnose ist die familiäre hypokalziurische Hyperkalzämie (FHH), die sehr selten mit relevanten Symptomen einhergeht und vor einer Nebenschilddrüsenoperation ausgeschlossen werden sollte $[9,10]$.

Idealerweise sollte ein $\mathrm{pHPT}$ auch vor jeglicher geplanten Schilddrüsenchirurgie ausgeschlossen werden, da ein erneuter Eingriff mit einem deutlich höheren Komplikationsrisiko verbunden wäre [11].

Die Diagnose wird biochemisch gestellt und ist zumeist einfach (Hyperkalzämie und erhöhtes Parathormon), die Lokalisation gestaltet sich jedoch manchmal schwierig. Die klinische Bedeutung des „normokalzämischen Hyperparathyreoidismus" ist noch relativ unklar, prinzipiell können aber auch hier die gleichen Effekte auf den Stoffwechsel erwartet werden wie beim asymptomatischen pHPT. Bei normalem Kalzium und erhöhtem Parathormon (PTH) ist die Differenzialdiagnose zum sekundären Hyperparathyreoidismus (HPT) sehr wichtig. Dies gelingt am besten dann, wenn eine Niereninsuffizienz, ein Vitamin-DMangel, eine gastrointestinale Erkrankung (Kalziummalabsorption) und eine Hyperkalziurie ausgeschlossen werden können. Betreffend die Notwendigkeit einer eventuellen Operation gelten beim normokalzämischen HPT laut Experten diegleichen Entscheidungsrichtlinien für ein invasives Vorgehen wie beim asymptomatischen pHPT.

Die Halssonografie kann ein Nebenschilddrüsenadenom (NSDA) häufig aufspüren, wobei dies naturgemäß auch von Patientencharakteristika wie den allgemeinen Schallbedingungen und vor allem der Erfahrung des Untersuchers abhängig ist. Im Ultraschall stellt sich ein
NSDA zumeist hypoechogen dar, obwohl normale Nebenschilddrüsen (NSD) isooder hyperechogen sind. Ein eindeutiger präoperativer Ultraschall ist für eine $\mathrm{Zu}$ weisung zur Parathyreoidektomie wohl ausreichend, wenn intraoperativ eine PTH-Bestimmung möglich und kein Verdacht auf eine genetische Ursache gegeben ist. Häufig wird man jedoch den Ultraschallbefund von einer Sestamibi-SPECT(Singlephotonen-Emissionscomputertomographie)-Untersuchung bestätigen lassen wollen. Singuläre Adenome sind in ca. $85-90 \%$ der Fälle die Ursache für den pHPT. Die Sensitivität für die Lokalisation multinodulärer NSDA ist sowohl mit der Sonografie als auch der Sestamibi-SPECT-Szintigrafie deutlich eingeschränkt.

Die Sestamibi-SPECT-Szintigrafie kann die verantwortliche Nebenschilddrüse bei solitären Adenomen zu 70-80\% lokalisieren, d.h. ein negativer Lokalisationsnachweis ist im klinischen Alltag nicht selten. Eine wesentliche Neuerung ist hier das Cholin-PET/CT, das sich seit wenigen Jahren in der klinischen Routine als sehr hilfreich herausgestellt hat und eine Sensitivität/Spezifität von $>90 \%$ hat $[12,13]$. In ausgewählten Einzelfällen kann auch ein (nur an wenigen Zentren verfügbares) MethioninPET erwogen werden [14].

Ob eine (in erster Linie chirurgische) Sanierung des pHPT empfohlen wird, ergibt sich aus Klinik und gezielten Untersuchungen. Alternativ ist bei ausgewählten Fällen eine Thermoablation (Radiofrequenzablation [RFA]) möglich [15]. Bei Patienten mit Kontraindikationen für 

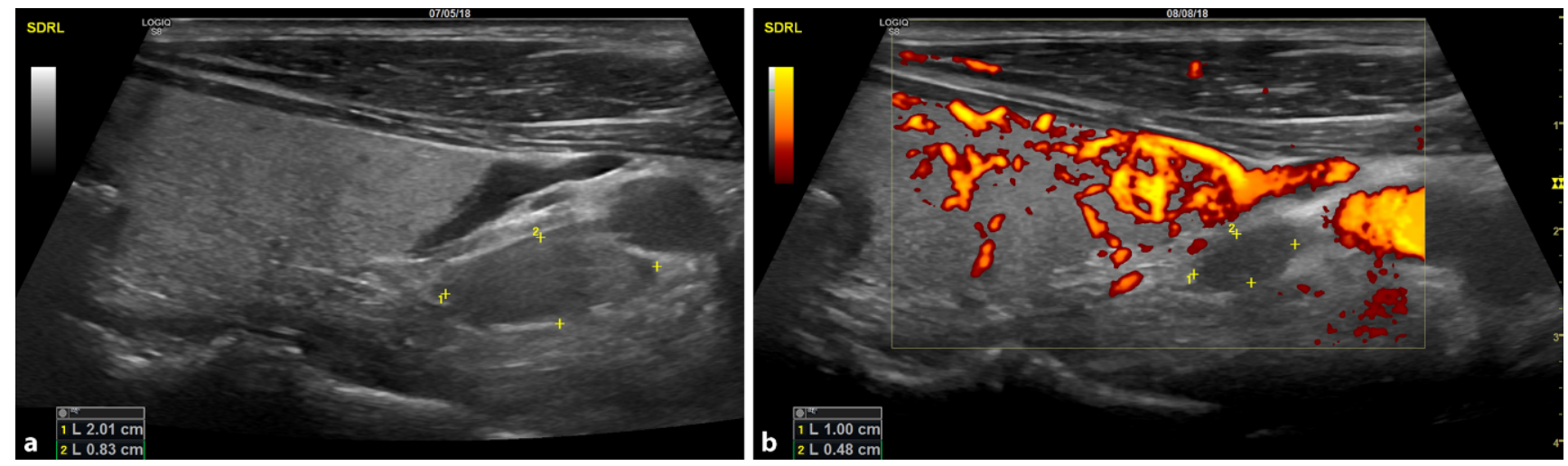

Abb. $1 \Delta$ a Fall 2. Dargestellt ist ein NSD-Adenom rechts kaudal, das Eiweiß-korrigierte Serumkalzium war mit 3,10 mmol/l, das PTH mit $139 \mathrm{pg} / \mathrm{ml}$ erhöht.Zehn Minuten nach abgeschlossener Radiofrequenzablation fiel das PTH auf $17 \mathrm{pg} / \mathrm{mlab}$. b Drei Monate nach der Radiofrequenzablation (RFA) hat sich das behandelte NSD-Volumen um $80 \%$ reduziert und entspricht in erster Linie einem avitalen Geweberest.Zehn Monate nach der Ablation sind alle relevanten Parameter weiterhin im Normbereich gelegen
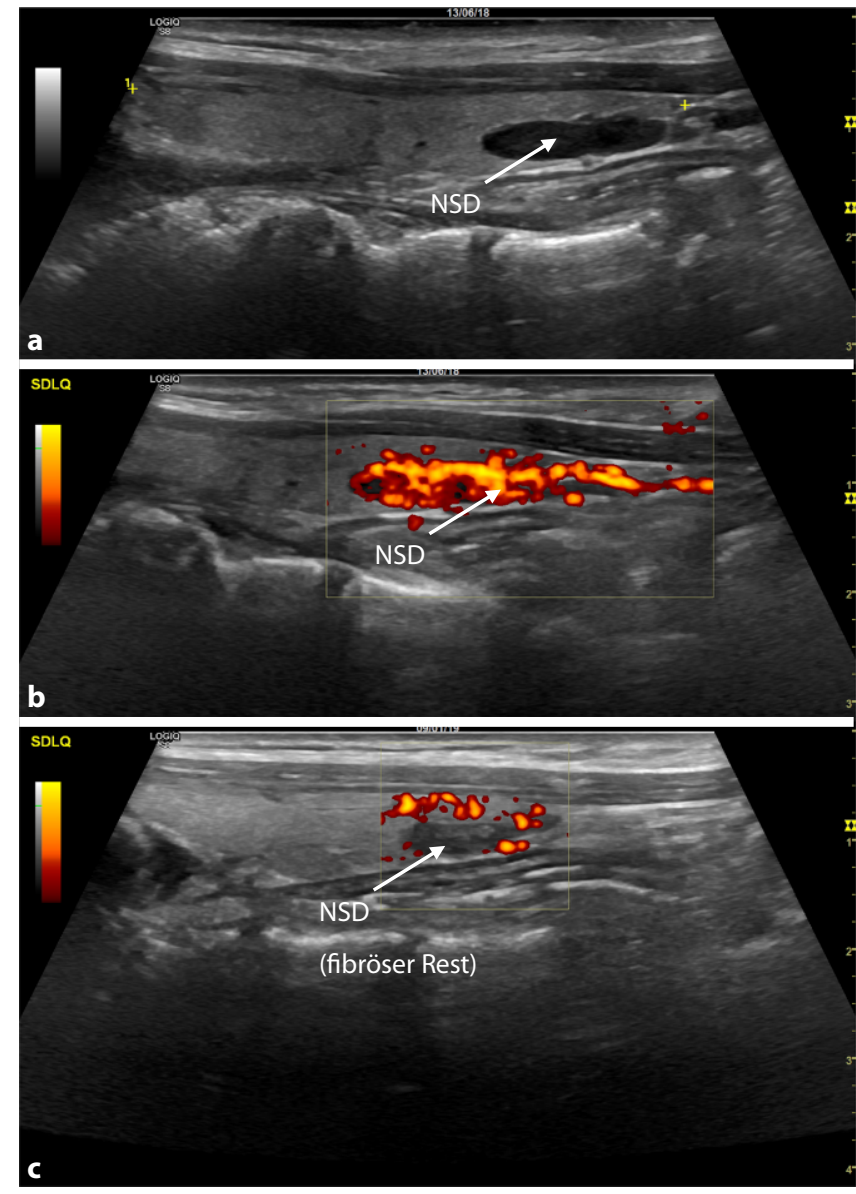

Abb. $2<$ a Langgestrecktes Nebenschilddrüsenadenom bei einer Patientin mit Status nach kranialer ipsilateraler Parathyreoidektomie vor einigen Jahren und kürzlichem Rezidiv einer Hyperkalzämie. Eine genetische Ursache konnte ausgeschlossen werden. $\mathbf{b}$ Gut darstellbare Hypervaskularisation des $\mathrm{Ne}$ benschilddrüsenadenoms. c Nach drei Monaten zeigt sich eine Volumsreduktion des NSD-Rests um $85 \%$ gegenüber dem Ausgangswert. Die "Devitalisierung" des NSD-Rests kommt durch die fehlende Vaskularisation gut zum Ausdruck

eine Parathyreoidektomie ist auch eine medikamentöse Therapie mit Cinacalcet (Mimpara) möglich [16, 17].

Eine retrospektive australische Studie bei Schwangeren mit pHPT zeigte eine relevante maternofetale Morbidität (v. a. Frühgeburtlichkeit und Präeklamp-

\section{Aktuelle Guidelines, Vitamin-D- und Kalziumzufuhr}

Die aktuellen Guidelines zur Entscheidungsfindung bei "asymptomatischem“ Hyperparathyreoidismus bezüglich des operativen Vorgehens wurden bereits 2014 publiziert. In der Vergangenheit kam es dabei immer wieder zu geringfügigen Adaptierungen. Diese Leitlinien sind allgemein sehr gut etabliert und in vielen Fällen eine große Entscheidungshilfe. Die Leitparameter für diese Beurteilung sind dabei Kalziumwerte, Frakturen, Nierenfunktion und Harnkalziumausscheidung ([19]; Tabelle 1).

In der Knochendichtemessung (Dual-X-Ray-Absorptiometrie, DXA) sollte daher neben der Radiusmessung zur Erfassung des kortikalen Knochens auch eine vertebrale Morphometrie zur Detektion von asymptomatischen Wirbelkörperfrakturen erfolgen. Auch das Harnkalzium sollte bestimmt werden (idealerweise im 24-h-Harn, eine NüchternSpotharnmessung ist jedoch eine gute Alternative).

Ein Vitamin-D-Mangel soll gesucht und mittels nativem Vitamin D korrigiert werden. Eine kalziumarme Ernährung ist obsolet - Kalzium soll bevorzugt über Ernährung zugeführt werden, nur bei Bedarf auch als Supplement [5, 9]. Bei relevanter Hyperkalziurie können Thiaziddiuretika ggf. kontrolliert versucht werden [20], obwohl diese auch als Risikofaktor für die Entstehung eines $\mathrm{pHPT}$ diskutiert werden [9]. 


\section{Fallvignetten}

\section{Fall 1}

Frau J. ist 27 Jahre alt, und seit einiger Zeit ist eine Schilddrüsenunterfunktion bei Hashimoto-Thyreoiditis bekannt. Es erfolgten auch mehrere Sonografien der Halsregion, in denen lediglich eine kleine Schilddrüse aufgefallen war. Seit etwa einem Jahr beklagte die Patientin Übelkeit sowie eine unspezifische Einschränkung des Wohlbefindens. In einer Laboruntersuchung zeigte sich eine Erhöhung des Serumkalziums $(2,80 \mathrm{mmol} / \mathrm{l})$, des PTH $(135 \mathrm{pg} / \mathrm{ml})$ sowie Harn-Spot-Kalziums $(0,77 \mathrm{mmol} / \mathrm{mmol})$. In der Knochendichtemessung (DXA) zeigt sich eine Osteopenie am Radius (T-Score -2,8), insgesamt besteht daher eine klare Operationsindikation.

Eine auswärtige NSD-Szintigrafie konnte kein Adenom identifizieren. Extern und auch bei uns war ein mehrfach gezielter Ultraschall negativ, worauf wir die Patientin für eine Cholin-PET/CT anmeldeten. In dieser konnte eindeutig ein NSDA rechts kaudaler Lokalisation gezeigt werden. Nach unkomplizierter Operation ist die Patientin biochemisch geheilt und berichtet über einen deutlich gebesserten Allgemeinzustand.

\section{Key Message}

Bei klassischer OP-Indikation und negativer Lokalisationsdiagnostik (Ultraschall und Sestamibi-SPECT-Untersuchung) ist ein Cholin-PET/CT ein oft hilfreicher nächster diagnostischer Schritt.

\section{Fall 2}

Frau H. ist 67 Jahre alt, und ein relevanter primärer Hyperparathyreoidismus sowie eine manifeste Osteoporose mit mehreren Wirbelkörperfrakturen (Th $6,7,8)$ sind seit Längerem bekannt. Zahlreiche weitere Erkrankungen (Diabetes mellitus Typ 2, Adipositas BMI 33, Vorhofflimmern unter NOAK, OSAS unter nächtlicher CPAP-Therapie) bestehen ebenfalls seit Jahren. Auswärts wurde eine Operation empfohlen, aufgrund der Komorbiditäten bestehtjedoch ein erhöhtes Narkoserisiko (zumindest

J. Klin. Endokrinol. Stoffw. 2019 · 12:50-54 https://doi.org/10.1007/s41969-019-0063-x (c) Der/die Autor(en) 2019

\section{K. Amrein · H. Dobnig}

Primärer Hyperparathyreoidismus. Drei spannende Fallberichte und Neuigkeiten zu Diagnose und Therapie

\section{Zusammenfassung}

Der primäre Hyperparathyreoidismus ( $p H P T)$ ist eine relativ häufige endokrine Erkrankung. Die Unterscheidung zwischen "asymptomatischem pHPT" und "asymptomatischem pHPT mit begleitenden, oft stummen Komplikationen" muss gründlich untersucht und herausgearbeitet werden, damit die beste weitere Vorgehensweise für den Patienten festgelegt werden kann. Die Standardtherapie bei symptomatischen Patienten ist die Operation, ebenso bei vielen Patienten mit asymptomatischem pHPT, wenn erfüllte Leitlinienkriterien ein solches
Vorgehen unterstützen. Mit dem Cholinund Methionin-PET(Positronen-EmissionsTomographie)/CT(Computertomographie) sowie der Radiofrequenzablation (nur in ausgewählten Fällen) haben sich in den letzten Jahren sowohl diagnostische als auch therapeutische Möglichkeiten zugunsten der Betroffenen weiter verbessert.

\section{Schlüsselwörter}

Primärer Hyperparathyroidismus · Radiofrequenzablation $\cdot$ Nebenschilddrüsenadenom Vitamin D.Cholin-PET

\section{Primary hyperparathyroidism. Three interesting case reports and innovations in diagnosis and therapy}

\section{Abstract}

Primary hyperparathyroidism (pHPT) is a frequent endocrine disorder. The difference between truly asymptomatic pHPT and asymptomatic pHPT with complications is often difficult and patients need to be examined extensively. For symptomatic patients and asymptomatic patients fulfilling current guideline criteria, standard therapy is surgery. In the last years, diagnosis and therapy have become more elegant with choline and methionine PET/CT for diagnosis and radiofrequency ablation for therapy in selected cases.

\section{Keywords}

Primary hyperparathyroidism · Radiofrequency ablation - Parathyroid adenoma Vitamin D . Choline PET
ASA 3), und die Patientin steht einer Operation sehr verhalten gegenüber. Laborchemisch zeigten sich ein erhöhtes Serumkalzium $(2,75 \mathrm{mmol} / \mathrm{l})$, PTH $(222 \mathrm{pg} / \mathrm{ml})$ sowie ein erniedrigtes $\mathrm{Vi}$ tamin D (16 ng/ml trotz 6000 IU D3/ Woche).

Da das NSDA topographisch günstig liegt, wurde der Patientin im Rahmen einer ausführlichen Aufklärung eine RFA angeboten. Das NSDA wurde mittels „PTH-Wash-out“ (Punktion und PTHBestimmung) gesichert (rechts kaudal, Größe $9 \times 4 \mathrm{~mm} ; 0,14 \mathrm{ml}$ Gesamtvolumen). Zehn Minuten nach komplikationsloser RFA zeigte sich ein PTH-Abfall von 220 auf $20 \mathrm{pg} / \mathrm{ml}$. In den Wochen darauf fiel klinisch trotz vermehrter Vitamin-D3- und Kalziumzufuhr ein leichtes Hungry-Bone-Syndrom auf. Drei Monate nach der RFA zeigte sich eine Volumenreduktion der behandelten NSD von $62 \%(0,06 \mathrm{ml}$, - Abb. 1a, b). Biochemisch waren die Laborwerte bei der letzten Kontrolle im Normbereich (Kalzium 2,46 mmol/l). Ein Beispiel für eine RFA eines kaudal gelegenen NSDA zeigt • Abb. 1.

\section{Key Message}

Bei relevanten Begleiterkrankungen und erhöhtem Anästhesierisiko sowie anatomisch günstigen Verhältnissen kann eine RFA in ausgewählten Fällen eine gute Alternative zu einer Operation sein.

\section{Fall 3}

Frau I. ist 55 Jahre alt und hatte 2014 eine Parathyreoidektomie links kranial, direkt postoperativ lagen die PTHund Kalziumwerte im Normbereich. 
Aufgrund eines erneuten Kalziumanstiegs im Juni 2018 wurde eine weitere Abklärung durchgeführt, hier zeigten sich ein PTH von $137 \mathrm{pg} / \mathrm{ml}$, ein Kalzium von $2,90 \mathrm{mmol} / \mathrm{l}$ bei gleichzeitig deutlich gesteigertem Knochenumbau (Osteokalzin 88,9 ng/ml) und normaler Nierenfunktion. Im Ultraschall zeigte sich ein intrathyreoidal gelegener hyperperfundierter Knoten suspekt auf ein NSDA, welches durch PTH-Washout bestätigt werden konnte ( $\bullet$ Abb. 2a). Eine genetische Ursache wurde durch eine molekularbiologische Analyse ausgeschlossen (MEN I/II und CDC73 negativ). Auch aufgrund des höheren Komplikationsrisikos bei Zweiteingriff $[11,21]$ entschied sich die Patientin für eine RFA. Zehn Minuten nach der Ablation war das PTH auf $16 \mathrm{pg} / \mathrm{ml}$ abgefallen. Über acht Wochen kam es $\mathrm{zu}$ einer temporären Heiserkeit und hypokalzämieassoziierten Muskelbeschwerden, die auf Kalziumsupplemente gut ansprachen. Drei Monate nach dem Eingriff zeigte sich eine Volumsreduktion der NSD von ca. $85 \%$, das Kalzium lag mit 2,44 mmol/l, PTH mit $41 \mathrm{pg} / \mathrm{ml}$ und Osteokalzin mit $30,8 \mathrm{ng} / \mathrm{ml}$ wieder im Normbereich, bzw. Letzteres war deutlich rückläufig. Durch die seltene intrathyreoideale Lage hätte chirurgisch eine Lobektomie links durchgeführt werden müssen, die aufgrund des kleinen zweiten Lappens $(<5 \mathrm{ml})$ praktisch sicher zu einer lebenslangen Schilddrüsenhormontherapie geführt hätte.

\section{Key Message}

Bei intrathyreoidalem NSDA kann eine RFA das gesunde Schilddrüsenparenchym erhalten.

\section{Cholin-PET/CT}

Wenn das Adenom sonografisch und szintigrafisch nicht lokalisiert werden kann, sollte ein Cholin-PET/CT erwogen werden. Dieses ist zwar kostspieliger, kann aber nahezu immer einen Lokalisationsnachweis erbringen [12, 13].

Eine grobe Kosten-Nutzen-Analyse ergab, dass eine A-priori-CholinPET/CT-Untersuchung bei allen Pati- enten wegen der negativen SestamibiBefunde bei rund $20-30 \%$ nicht viel teurer kommt als das bisherige diagnostische Vorgehen [22]. Dazu kommt, dass nach Cholin-PET/CT-Lokalisationsdiagnostik mit großer Wahrscheinlichkeit auf eine intraoperative PTH-Bestimmung verzichtet werden könnte, was sich in einer Kosten-Nutzen-Analyse ebenfalls positiv niederschlagen würde.

\section{RFA bei}

\section{Hyperparathyreoidismus}

Die definitive Sanierung eines primären Hyperparathyreoidismus sollte operativ erfolgen. Neben dem klassischen chirurgischen Eingriff kann bei ausgewählten Fällen auch eine Radiofrequenzablation erwogen werden. Dafür muss in erster Linie das Nebenschilddrüsenadenom gewisse Kriterien erfüllen (Identifizierung, allgemeine Topografie und Lage zu Gefäßen/N. recurrens, Größe). Vorteile inkludieren das interventionelle Vorgehen in Lokalanästhesie, die ambulante Behandlung und die sehr kurze Rekonvales-

Hier steht eine Anzeige. 
zenz. Gerade für ältere oder polymorbide Menschen mit hohem Narkose-/OPRisiko kann dies einen großen Vorteil bedeuten.

\section{Korrespondenzadresse}

PD Dr. Karin Amrein, MSc

Schilddrüsen/Endokrinologie/Osteoporose Institut Dobnig

Graz, Österreich

karin.amrein@medunigraz.at

Funding. Open access funding provided by Medical University of Graz.

\section{Einhaltung ethischer Richtlinien}

Interessenkonflikt. K. Amrein und H. Dobnig geben an, dass kein Interessenkonflikt besteht.

Alle beschriebenen Untersuchungen am Menschen oder an menschlichem Gewebe wurden mit Zustimmung der zuständigen Ethikkommission, im Einklang mit nationalem Recht sowie gemäß der Deklaration von Helsinki von 1975 (in der aktuellen, überarbeiteten Fassung) durchgeführt. Von allen beteiligten Patienten liegt eine Einverständniserklärung vor.

Open Access. Dieser Artikel wird unter der Creative Commons Namensnennung 4.0 International Lizenz (http://creativecommons.org/licenses/by/4.0/deed. de) veröffentlicht, welche die Nutzung, Vervielfältigung, Bearbeitung, Verbreitung und Wiedergabe in jeglichem Medium und Format erlaubt, sofern Sie den/die ursprünglichen Autor(en) und die Quelle ordnungsgemäß nennen, einen Link zur Creative Commons Lizenz beifügen und angeben, ob Änderungen vorgenommen wurden.

\section{Literatur}

1. Asban A, Dombrowsky A, Mallick R, Xie R, Kirklin JK, Grogan RH et al (2019) Failure to diagnose and treat Hyperparathyroidism among patients with hypercalcemia: opportunities for intervention at the patient and physician level to increase surgical referral. Oncologist pii:theoncologist.2018-0424. https://doi.org/10 1634/theoncologist.2018-0424

2. Bilezikian JP, Cusano NE, Khan AA, Liu JM, Marcocci C, Bandeira F (2016) Primary hyperparathyroidism. Nat Rev Dis Primers 2:16033

3. Rolighed L, Amstrup AK, Jakobsen NF, Sikjaer T, Mosekilde L, Christiansen P et al (2014) Muscle function is impaired in patients with "asymptomatic" primary hyperparathyroidism. World J Surg 38(3):549-557

4. Selberherr A, Hormann M, Prager G, Riss P, Scheuba C, Niederle B (2017) "Silent" kidney stones in "asymptomatic" primary hyperparathyroidism - a comparison of multidetector computed tomography and ultrasound. Langenbecks Arch Surg 402(2):289-293

5. Bilezikian JP (2018) Primary Hyperparathyroidism. JClin Endocrinol Metab 103(11):3993-4004
6. Silva BC, Cusano NE, Bilezikian JP (2018) Primary hyperparathyroidism. Best Pract Res Clin Endocrinol Metab 32(5):593-607. https://doi.org/10. 1016/j.beem.2018.09.004

7. Cetani F, Saponaro F, Borsari S, Marcocci C (2019) Familial and hereditary forms of primary hyperparathyroidism. Front Horm Res 51:40-51

8. Ruda JM, Hollenbeak CS, Stack BC Jr. (2005) A systematic review of the diagnosis and treatment of primary hyperparathyroidism from 1995 to 2003. Otolaryngol Head Neck Surg 132(3):359-372

9. Bilezikian JP, Bandeira L, Khan A, Cusano NE (2018) Hyperparathyroidism. Lancet 391(10116):168-178

10. Bhangu JS, Selberherr A, Brammen L, Scheuba C, Riss P (2019) Efficacy of calcium excretion and calcium/creatinine clearance ratio in the differential diagnosis of familial hypocalciuric hypercalcemia and primary hyperparathyroidism. Head Neck 41(5):1372-1378

11. Medas F, Tuveri M, Canu GL, Erdas E, Calò PG (2019) Complications after reoperative thyroid surgery: retrospective evaluation of 152 consecutive cases. Updates Surg. https://doi.org/10.1007/s13304019-00647-y

12. Broos WAM, van der Zant FM, Knol RJJ, Wondergem M (2019) Choline PET/CT in parathyroid imaging: a systematic review. Nucl Med Commun 40(2):96-105

13. Treglia G, Piccardo A, Imperiale A, Strobel K, Kaufmann PA, Prior J, Giovanella L (2018) Diagnostic performance of choline PET for detection of hyperfunctioning parathyroid glands in hyperparathyroidism: a systematic review and meta-analysis. Eur J Nucl Med Mol Imaging 46(3):751-765. https://doi.org/10.1007/s00259018-4123-z

14. Møller ML, Rejnmark L, Arveschoug AK, Højsgaard A, Rolighed L (2018) Clinical value of 11Cmethionine positron emission tomography in persistent primary hyperparathyroidism-a case report with a mediastinal parathyroid adenoma. Int J Surg Case Rep 45:63-66

15. Machi J (2006) Radiofrequency ablation for hyperparathyroidism: can it be a new treatment? Surg Laparosc Endosc Percutan Tech 16(2):116

16. Khan A, Bilezikian J, Bone H, Gurevich A, Lakatos $P$, Misiorowski W et al (2015) Cinacalcet normalizes serum calcium in a double-blind randomized, placebo-controlled study in patients with primary hyperparathyroidism with contraindications to surgery. Eur J Endocrinol 172(5):527-535

17. Schwarz P, Body J, Cap J, Hofbauer LC, Farouk M, Gessl A et al (2014) The PRIMARA study: A prospective, descriptive, observational study to review cinacalcet use in patients with primary hyperparathyroidism in clinical practice. Eur J Endocrinol 171(6):727-735

18. Rigg J, Gilbertson E, Barrett HL, Britten FL, Lust $\mathrm{K}$ (2019) Primary hyperparathyroidism in pregnancy: maternofetal outcomes at a quaternary referral obstetric hospital, 2000 through 2015. J Clin Endocrinol Metab 104(3):721-729

19. Bilezikian JP, Brandi ML, Eastell R, Silverberg SJ, Udelsman R, Marcocci Cet al (2014) Guidelines for the management of asymptomatic primary hyperparathyroidism: summary statement from the Fourth International Workshop. J Clin Endocrinol Metab 99(10):3561-3569

20. Riss $P$, Kammer M, Selberherr A, Bichler $C$, Kaderli $R$, Scheuba C et al (2016) The influence of thiazide intake on calcium and parathyroid hormone levels in patients with primary hyperparathyroidism. Clin Endocrinol (Oxf) 85(2):196-201
21. Bai B, Chen Z, Chen W (2018) Risk factors and outcomes of incidental parathyroidectomy in thyroidectomy: a systematic review and metaanalysis. PLoS ONE 13(11):e207088

22. Burger I (2017) Cholin PET bei Nebenschilddrüsenadenom. https://www.svmtra. ch/files/Dokumente/Verband/Fachstellen/ Nuklearmedizin/Refresh_yourself_2017/8_ 171030 Burger_Irene_A._Cholin_PET_beim Nebenschilddruesenadenom.pdf. Zugegriffen: 24. Mai 2019

Hinweis des Verlags. Der Verlag bleibt in Hinblick auf geografische Zuordnungen und Gebietsbezeichnungen in veröffentlichten Karten und Institutsadressen neutral. 